\title{
Barriers and incentives to recruitment in mental health clinical trials
}

\author{
Helen Jones, Andrea Cipriani \\ Department of Psychiatry, Warneford Hospital, University of Oxford, Oxford, UK; Oxford Health NHS \\ Foundation Trust, Warneford Hospital, Oxford, UK
}

Corresponding author:

Professor Andrea Cipriani,

Department of Psychiatry,

Warneford Hospital,

University of Oxford,

Oxford OX3 7JX,

UK

Conflict of Interest: none

Acknowledgements: Andrea Cipriani is supported by the National Institute for Health Research

(NIHR) Oxford Health Biomedical Research Centre (grant BRC-1215-20005), by an NIHR Research

Professorship (grant RP-2017-08-ST2-006) and by the NIHR Oxford Cognitive Health Clinical Research Facility. The views expressed are those of the authors and not necessarily those of the UK National Health Service, the NIHR, or the UK Department of Health. 
Research provides valuable information that improves patients' outcomes and should inform clinical decision making. ${ }^{1}$ There are many research methodologies ${ }^{2}$ and randomised controlled trials (RCTs) are at the top of the hierarchy, providing the most robust results when efficacy of interventions is concerned. ${ }^{3}$ RCTs are considered the gold standard because randomisation is the best method we have to remove selection bias between two groups of patients. However, to randomise participants in a trials, there has to be genuine uncertainty in the clinicians over whether a treatment will be beneficial. ${ }^{4}$ This is called clinical equipoise, which provides the ethical basis for medical research that involves assigning patients to different treatment arms of a clinical trial. ${ }^{5}$

Notwithstanding all these considerations, it is well recognised that many RCTs struggle to recruit an adequate sample size, with large, collaborative studies often not being able to meet the targets as originally planned. Difficulty in recruitment may result in a series of problems (e.g. research samples unrepresentative of the intended study population, or reduced statistical power that can affect validity of research findings. ${ }^{6}$

The published literature recognises a number of the barriers to recruitment in clinical studies: ${ }^{7,8}$

- Clinicians' difficulties with understanding equipoise and their confidence in expressing this concept to potential trial participants;

- Clinicians' application of exclusion criteria independent of the study protocol;

- Gatekeeping behaviours, based on assumptions that participation in trial may be too stressful;

- Concern that research involvement may potentially damage patient-clinician relationships;

- Time and workload pressures, and competing clinical priorities for clinical staff;

- Negative attitudes towards research and evidence-based practice;

- Lack of confidence in explaining research to participants;

- Discrepancies between "policy" and "practice", for example revalidation process requires medical staff to provide evidence of research work, however there is no protected time or funds in clinical work to facilitate this. 
To overcome potential barriers to recruitment, interventions to improve recruitment have been examined in individual studies and also in reviews, including the following: ${ }^{9}$

- Feedback to the trial clinicians based on recorded and transcribed data of recruitment appointments, with identification of time spent on explaining trial design, treatment arms, procedures and total length of appointment.

- Protected research time and extra training.

- Reduction in clinicians' workload and time to recruit.

- Training, including communications skills and role play.

If all this is true for medicine in general, recruitment into clinical trials in populations suffering from mental illness face additional challenges, often related to perceived patient vulnerability and preferences of professionals and patients preferences, which can affect the decision regarding randomisation of a patient and their participation in a treatment arm of a study. ${ }^{10}$ Furthermore, in mental health settings treatment decisions are frequently at crisis points when patients are disturbed or distressed, and when capacity to consent to treatment, and perceived capacity to take on new information by potential participants may be limited. ${ }^{11}$

Researcher's awareness of the barriers to recruitment to mental health RCTs is increasing and data have been published to illustrate the difficulties experienced by researchers in trying to reach preplanned sample sizes, and its influence on trial results. Previous reviews on barriers to recruitment have not included evidence from mental health trials, such as relevant data from the CUtLASS trial. ${ }^{12}$ Furthermore, although data exist that indicate that qualitative interventions may be effective in improving clinicians' confidence to recruit to RCTs, ${ }^{13}$ there is no clear mention of data specific to mental health settings.

This is an area that deserves further and systematic exploration and analysis. We need to better understand the barriers and incentives to recruitment in mental health trials and guide clinical 
researchers and policy makers in the development of interventions that may help improve clinician's readiness to approach, recruit and randomise patients into RCTs. Improving patients outcomes and transforming NHS services by generation of good quality research is also set out as one of the priorities by NHS England (https://www.england.nhs.uk/wp-content/uploads/2017/04/nhseresearch-plan.pdf). 


\section{References}

1. Tomlinson A, Cipriani A. The times they are a-changin'. Evid Based Ment Health 2018;21:81.

2. Leucht S, Chaimani A, Cipriani A, et al. Network meta-analyses should be the highest level of evidence in treatment guidelines. Eur Arch Psychiatry Clin Neurosci. 2016;266:477-80.

3. Furukawa TA, Cipriani A, Leucht $\mathrm{S}$, et al. Is placebo response in antidepressant trials rising or not? A reanalysis of datasets to conclude this long-lasting controversy. Evid Based Ment Health. 2018;21:1-3.

4. Kendall T, Morriss R, Mayo-Wilson E, et al. Assessment and management of bipolar disorder: summary of updated NICE guidance. BMJ 2014;349:g5673.

5. Cook C, Sheets $C$. Clinical equipoise and personal equipoise: two necessary ingredients for reducing bias in manual therapy trials. J Man Manip Ther 2011;19:55-7.

6. Rendell JM, Merritt RK, Geddes J. Incentives and disincentives to participation by clinicians in randomised controlled trials. Cochrane Database Syst Rev 2007;2:MR000021.

7. Donovan JL, de Salis I, Toerien M, et al. The intellectual challenges and emotional consequences of equipoise contributed to the fragility of recruitment in six randomized controlled trials. J Clin Epidemiol 2014;67:912-20.

8. Borschmann R, Patterson S, Poovendran $D$, et al. Influences on recruitment to randomised controlled trials in mental health settings in England: a national cross-sectional survey of researchers working for the Mental Health Research Network. BMC Med Res Methodol 2014;14:23.

9. Paramasivan $\mathrm{S}$, Strong $\mathrm{S}$, Wilson $\mathrm{C}$, et al. A simple technique to identify key recruitment issues in randomised controlled trials: Q-QAT - Quanti-Qualitative appointment timing. Trials 2015;16:88.

10. Howard L, de Salis I, Tomlin Z, et al. Why is recruitment to trials difficult? An investigation into recruitment difficulties in an RCT of supported employment in patients with severe mental illness. Contem Clin Trials 2009;30:40-6.

11. Morant N, Kaminskiy E, Ramon S. Shared decision making for psychiatric medication management: beyond the micro-social. Health Expect 2016;19:1002-14.

12. Jones PB, Barnes TR, Davies L, et al. Randomized controlled trial of the effect on Quality of Life of second- vs first-generation antipsychotic drugs in schizophrenia: Cost Utility of the Latest Antipsychotic Drugs in Schizophrenia Study (CUtLASS 1). Arch Gen Psychiatry 2006;63:1079-97. 
13. Townsend D, Mills, N, Savović J, et al. A systematic review of training programmes for recruiters to randomised controlled trials. Trials 2015;16:432. 